\title{
Simulation study of load sensing dynamics on hydraulic excavator
}

\author{
Ken Ichiryu, Masami Ochiai, Tsukasa Toyooka \\ Technical Research Laboratory, Hitachi Construction Machinery. Co. \\ Tsutiura-shi Kandatsu 650, Japan \\ Kazunori Nakamura \\ Tsuchiura Works, Hitachi Construction Machinery. Co. Ibid
}

\section{ABSTRACT}

Load sensing concept and general control scheme are not new and it has been widely applied for may industrial hydraulic systems.

But, for the application of hydraulic excavator, there remains many technical problems to overcome, such as vibration due to load sensing servo loop, acceleration control of inertia load and maneuverability of simultaneous multioperation of actuators.

We constructed simulator model of the hydraulic excavator.

Most difficult problem of load sensing is how to suppress instability due to low damping of the load sensing dynamics.

Secondary difficult problem is how to obtain smooth operation for inertia load. We studied these problems by simulating the detailed behavior of load sensing hydraulic system, and obtained good agreement with the experiment.

By combining the above mentioned simulator study, we could newly develop electronically controlled load sensing hydraulic excavator.

1. Load sensing system of hydraulic excavator.

1.1 Present state of load sensing system.

General load sensing system (LS) configuration is shown in fig. 1 .

LS system means that by detecting actuator's maximum load and controlling pump delivery pressure certain value higher than the detected maximum load pressure, to prevent excess energy loss and to keep higher circuit efficiency.

In case of 1 pump system equipped with multiple actuators as shown in the figure, LS system controls variable displacement pump to keep differential pressure constant (LS differential pressure $\triangle P_{L S}$ ) by the action of $L S$ valve by detecting circuit maximum load pressure.
Because, parallel circuit is formed for each actuator, input flow for each actuator fluctuates in combined operation.

To prevent above phenomena, pressure compensators (PC) are installed which function to keep constant of the differential pressure between inlet and outlet of each flow control valve.

Besides, unload valve is equipped which operates under the differential pressure between pump and maximum load pressure $\triangle \mathrm{PLS}$, and its set differential pressure is adjusted higher than command LS differential pressure $\triangle$ PLSC, thus reduction of flow energy loss of pump minimum inclination is achieved at the all flow control valves neutral position. 
By these arrangements, each actuator's independence is kept and superior combined operatorbility is achieved.

LS is a system which controls pump delivery flow rate according to demand load flow rate passing through flow control valve, therefore, especially efficient at single operation, because pump energy is nearly equal to load energy and loss energy is kept considerably small.

On the contrary, as LS system constitutes pressure servo loop, it receives the influence of load characteristics more directly, and increases instability, because fundamental nature of differential pressure constant control brings about low damping.

We could say briefly LS system is apt to become dynamically unstable.

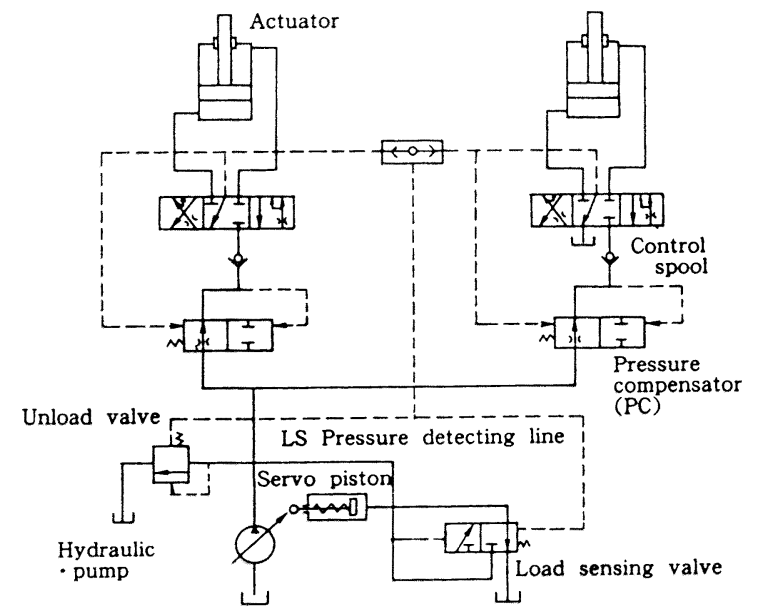

Fig.1 General load sensing system

\subsection{New LS excavator system}

Newly developed hydraulic excavator system is shown in fig.2. This system consists of electronically controlled 1 pump load sensing system and is controlled by $\mu$-processor.

$\mu$-processor control includes pump, valve and engine, etc.

Pump delivery pressure, pump inclination angle, and pump rotational speed are sensed electronically, also LS differential pressure $\triangle P L S$ is sensed electronically by newly developed sensing device.

Pump inclination angle is governed by two high speed on-off solenoids, and pump control includes LS, speed sensing-torque absorbing scheme.

Brief explanation of pump control is shown in fig.3. Command inclination angle $\theta_{\mathrm{C}}$ is determined by the minimum selection of $\theta_{\mathrm{LS}}$ and $\theta \mathrm{t}$, where $\theta_{\mathrm{LS}}$ is inclination target determined by LS control which is a function of load sensing differential pressure $\triangle \mathrm{PLS}_{\mathrm{L}}$ from the differential sensor, command differential pressure $\triangle \mathrm{PLSC}$, and present inclination angle $\theta_{0}$, and $\theta t$ is torque control target inclination determined by pump delivery pressure Ps and engine rotational speed $\mathrm{N}$.

DE shown in fig. 3 is a dead band of pump inclination control and this value is determined by compromise of stability and exactness of control.

Furthermore, there is a matching mode which changes response speed according to working modes.

Each flow control valve has a variable pressure compensator which controls compensating value from outer signal pressure.

This variable PC is governed via proportional solenoid from the controller signal, therefore it is possible to set proper compensating pressure best fit for each circumstance.

In saturated condition, by detecting $\triangle \mathrm{P}_{\text {LS }}$ reduction value, controller determines set value of $P C$ and supervises

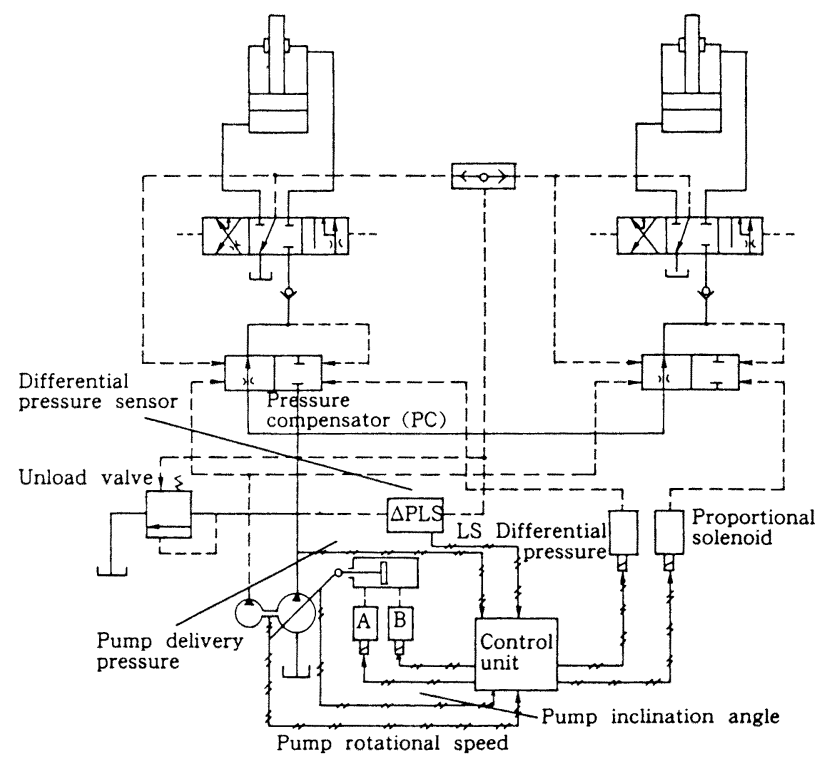

Fig.2 Newly developed load sensing hydraulic excavator (Hitachi) 
variable pressure compensator.

By this control good combined operation becomes possible in a saturated condition. Further, setting of PC corresponding to working mode and adjusting of response of PC make easy operation possible.

\section{Simulation model}

Simulation analysis using continuous simulation language ACSL (Advanced Continuous Simulation Language) was performed, for the purpose of (i) understanding of influence degree of each LS system component's parameter on stability (ii) development of stable and easily operative excavator.

Fig.4. shows construction of hydraulic excavator. Simulation analyses consist of two programs. Program (A) treats mainly single operation of boom, whose model simulates real machine configuration, and by this program each component parameter's influence on stability is studied, extensively.

Calculation time mesh is $1 \mathrm{~ms}$ for hydraulics and load system and $5 \mathrm{~ms}$ for control system, respectively.

Pump regulator is modeled by on-off servo which uses high speed on-off solenoid. LS and pump pressure signal 1ines are modeled by 1 st order delay. PC dynamics is also expressed by 1st order delay system.

Simulation program (B) treats mainly dynamic response under combined operation and dynamic behavior of inertia load system, such as swing and travel system. In this case multi actuators are simulated simultaneously.

Outline of this block diagram is shown in $\mathrm{fig} .5$.

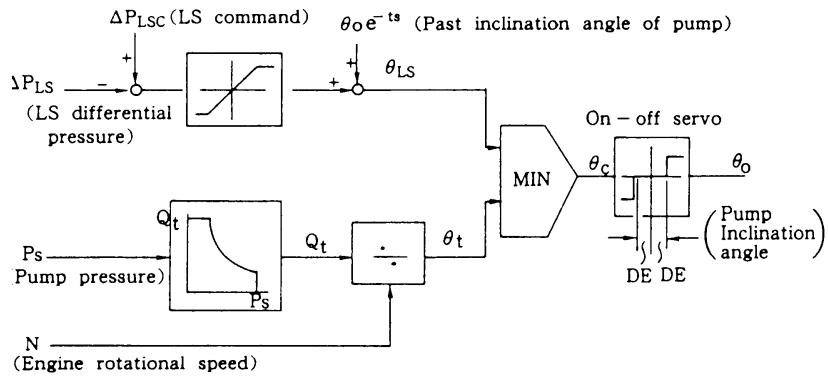

Fig.3 Brief explanation of pump control
At this program, interference of each actuator is neglected. Speed sensing control is attached to pump control in this program. The other parts are same with program $(A)$.

3. Stability of load sensing system

3.1 Stability effect of piping and pump regulator parameters

Analysis was performed to know what kind of effect influences the above parameters on the stability of LS system, under the condition without operation of pressure compensator, at the $\triangle \mathrm{P}_{\mathrm{LS}}=1.5 \mathrm{MPa}$ and $\mathrm{DE}=0.15 \mathrm{MPa}$.

By this study, it was clarified that (a) pump line volume reduction (b) shortening of pump signal delay (C) enlargement of meter-in volume, contribute to stability.

That is, piping design is desirable whose pump pressure signal response is high, and load pressure signal response has appropriate delay.

For example, influence of pump line volume is shown in fig.6. Comparing $\mathrm{Vp}=1.8 \ell$ case, with $\mathrm{Vp}=4.0 \ell$ case, it is clear that the former vibration decays more rapidly than the latter, and stability is improved considerably.

Pump regulator uses on-off servo to give high response.

In that case, regulator receives self excited high frequency vibration when dead band is small, but by widening dead band $\mathrm{DE}$ to $0.2 \mathrm{MPa}$, system becomes stable.

LS differential pressure (diff.pr) is determined by throtting loss of flow control valve. LS diff.pr fluctuation to pump delivery pressure change becomes large at small flow rate than at large flow rate. This corresponds to the fact that at the fine operation machine is apt to become unstable, this agreement supports validity of simulator.

To make DE excessively large is not a good thing, because it worsens control quality.

So that in actual case, the value is changed according to working condition. Unload valve is fundamentally an element which unloads minimum flow rate at pump neutral condition. 


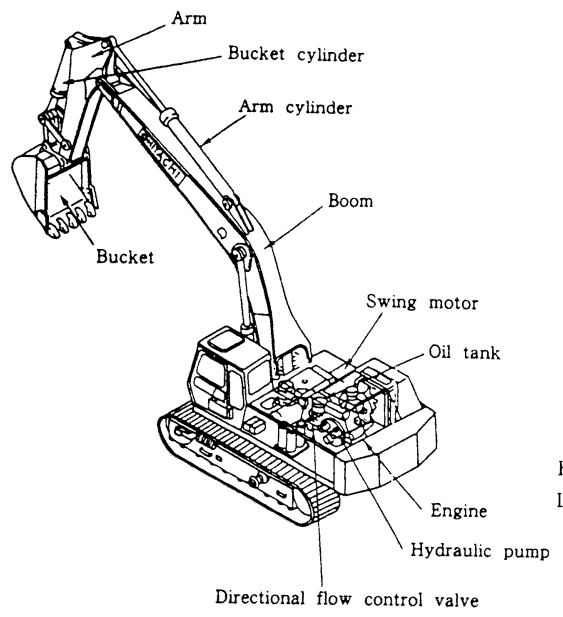

Directional flow control valve

Fig. 4 Costruction of hydraulic excavator

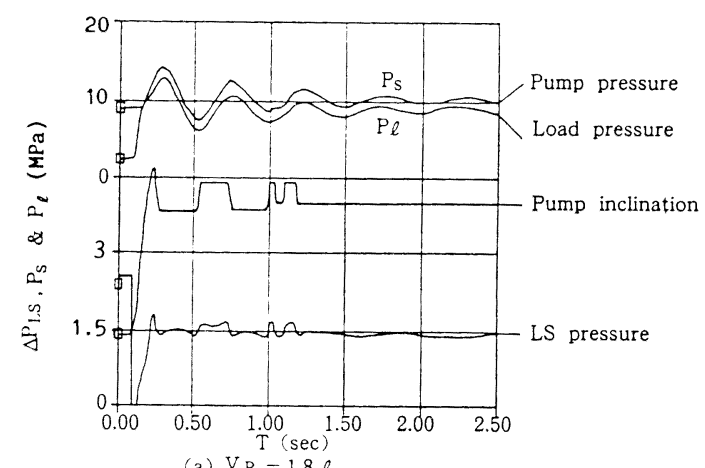

(a) $\mathrm{VP}=1.8 \mathrm{\ell}$

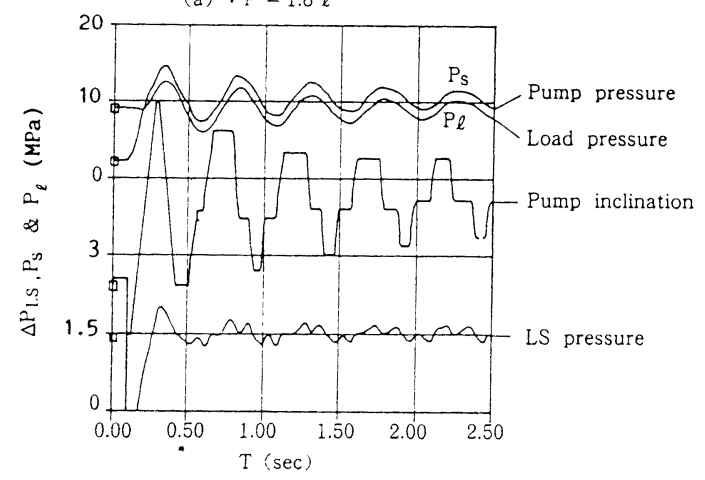

(b) $V P=4.0 \ell$

Fig. 6 Influence of pump line volume

We considered to achieve LS system stability by the extension of this nature. Improved unload valve's characteristics, as shown in fig.7. is to give pre-leakage at the normal $\triangle \mathrm{PLS}_{\mathrm{LS}}$ condition, in other word, when $\triangle \mathrm{PLS}$ is same with command signal.

Fig.8. (a)(b) are simulated results of leakage effect of unload valve at boom rise operation, the former (a) corresponds to conventional unload valve without pre-leakage. Severe vibration is observed.
Engine speed sensing effect

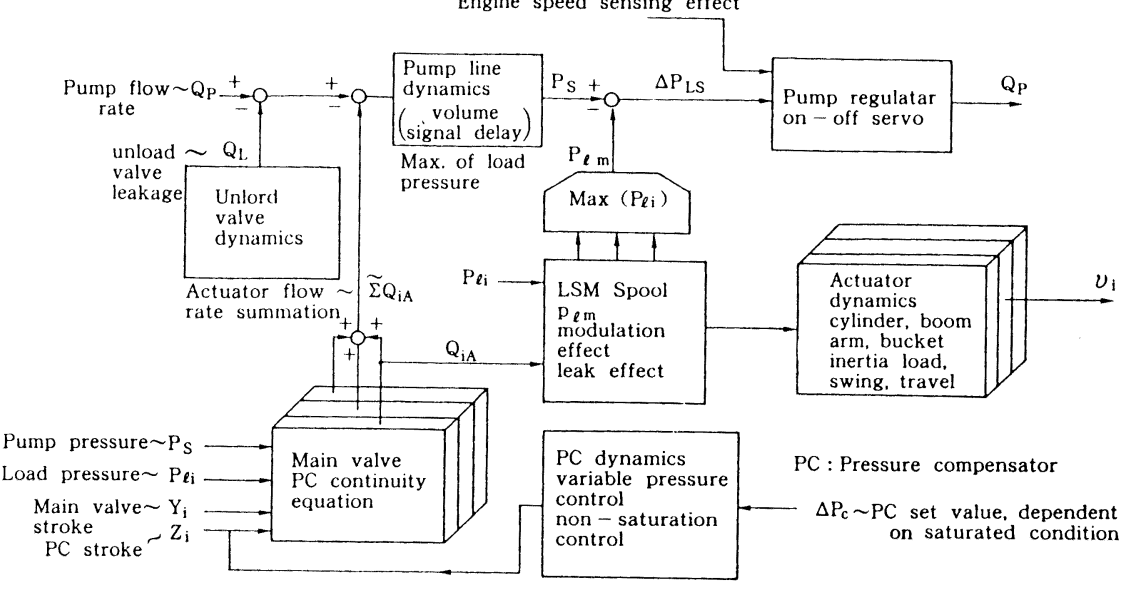

Fig.5 Outline of the simulator block diagram

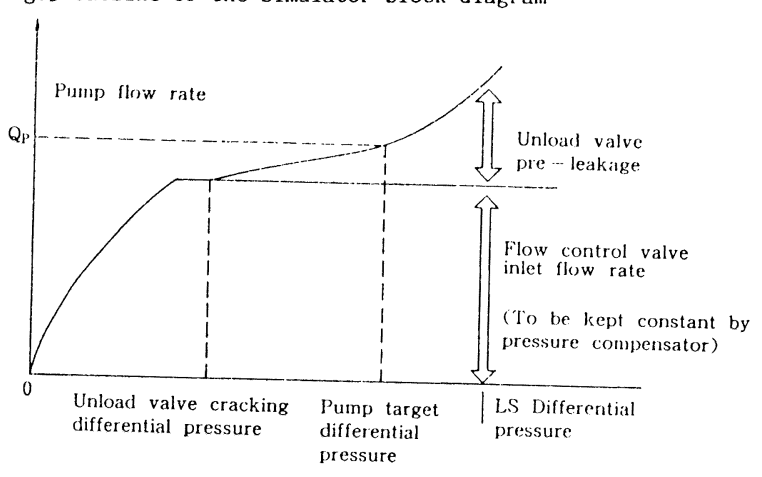

Fig.7 Unload valve characteristics

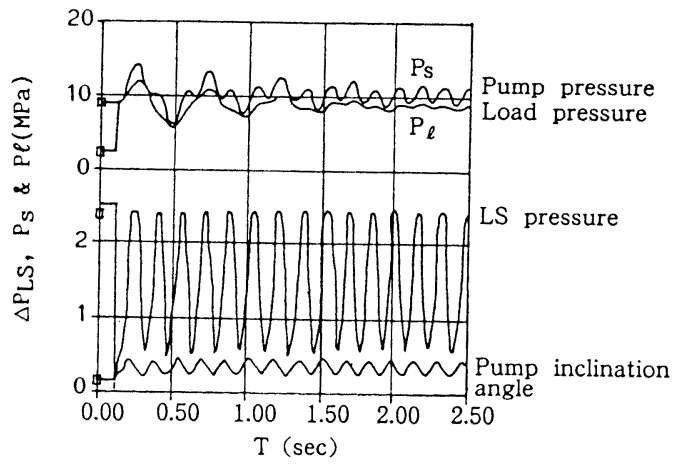

(a) Conventional unload valve (no pre-leakage)

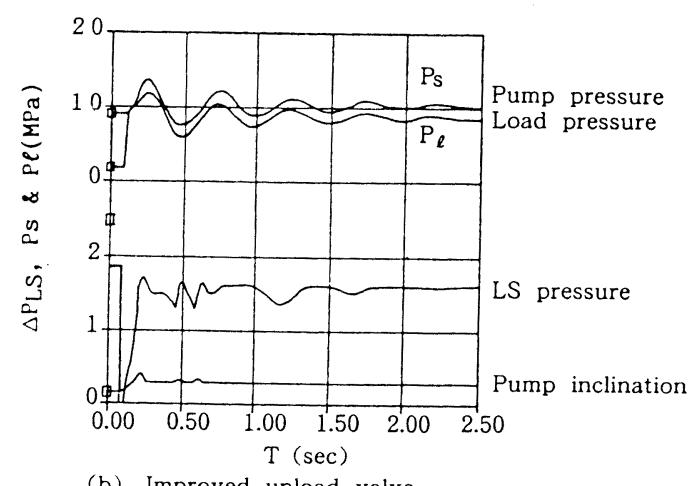

(b) Improved unload valve (pre-leakage)

Fig. 8 Effect of unload valve pre-leakage on boom rise 
On the contrary, fig.(b) is the case of improved unload valve with preleakage, severe vibration is prevented and stable operation becomes possible. This effect is confirmed in boom rise experiment of hydraulic excavator.

\subsection{Improvement of dynamic characteris-} tics of inertia load system.

In case of pure inertia load system such as swing and travel at is control, even by inching operation operating pressure reaches relief pressure level and acceleration force becomes too large. Therefore, operating feeling of inching becomes worse, in some case. For this improvement, we devised LSM (Load Sensing Modulated) Spool, whose function is to control load's maximum pressure depending on lever stroke.

By this device, smooth inching operation was realized.

This device bleeds small amount of flow from LS signal line to tank, and makes LS signal by reducing load pressure corresponding to spool stroke.

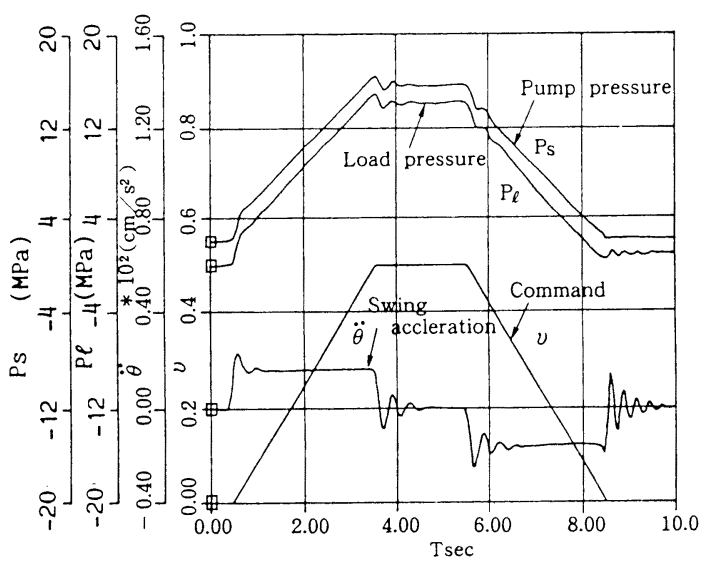

(a) Conventional spool

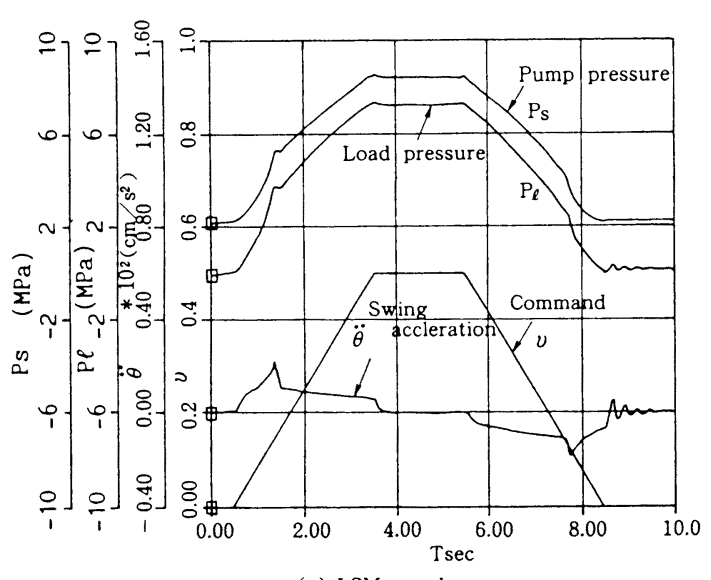

(a) LSM spool

Fig.10 Effect of LSM spool on swing motion
Comparison of static characteristics between conventional and LSM spool is shown in fig.9. In the conventional type, pressure change versus stroke is very sharp, but in the LSM spool pressure changes slowly versus stroke.

In other words, LSM spool, by making pressure gain $(\Delta p / \Delta \chi)$ of spool small, gives damping to the system and changes its function from flow control to pressure control.

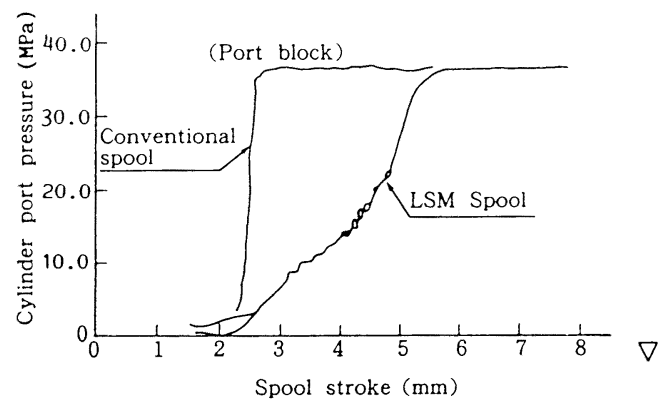

Fig. 9 Comparison between conventional and LSM spool characteristics

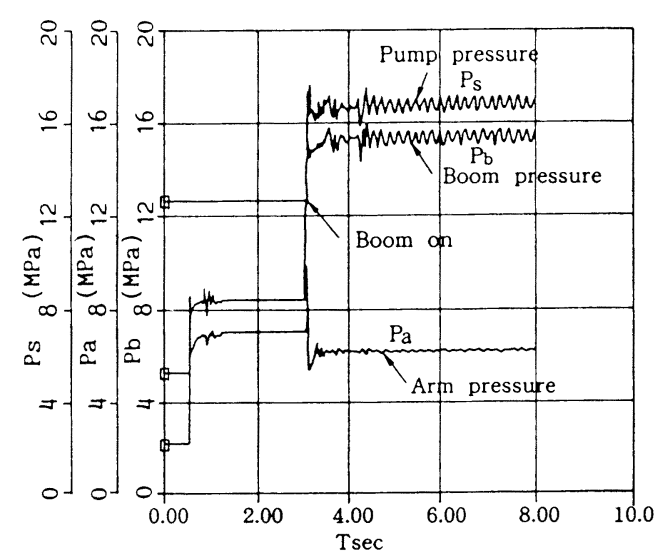

(a) Conventional design of PC $\beta=0$

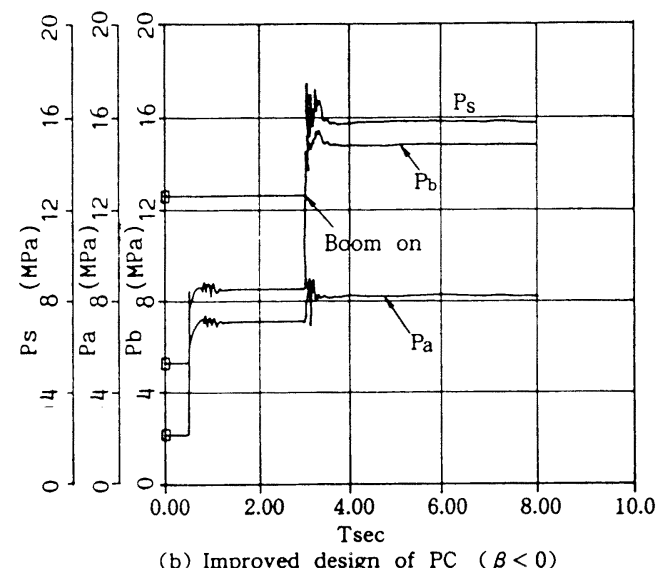

(b) Improved design of PC $(\beta<0)$

Fig. 11 Unstable phenomena of combined operation (Combined operation, boom rapid operation)
PC : pressure compensator 
In fig. 10. comparison of simulation results between conventional and LSM spool is shown. This simulation supposes swing fine operation whose input is ramp, 3 second from zero to half stroke.

In case of conventional spool, actuator receives sudden acceleration regardless of fine operation.

But in case of LSM spool, actuator's acceration and deceleration are understood quite smooth.

Also at the acceleration and deceleration stage, conventional spool has tendency of hunting, but LSM spool is stable without sign of hunting.

This implies that in LSM spool damping effect is achieved by bleeding small flow rate and LSM spool is quite effective for hunting countermeasures.

\subsection{Unstable phenomena of combined op-} eration.

As one countermeasure for hunting of large load actuator operation, such as boom rise, leak effect of unload valve was discussed in previous paragraph.

This hunting phenomenon is considered caused by inherent characteristics of PC for the fluctuation of load pressure.

When cylinder load is considered, damping for the system is given when pressure gain of $\mathrm{PC}, \beta=\mathrm{dQc} / \mathrm{dPc}$ is negative (where $Q_{C}$ : cylinder inlet flow rate, $P_{C}$ : cylinder pressure). That is, if $Q_{C}$ shows right side downward characteristic for the self pressure $P_{C}$, system becomes stable.

New system enables combined operation by flow dividing by means of controlling set pressure of $\mathrm{PC}$, under saturation state.

In these miscellaneous combined mode experiments, typical hunting phenomena were observed for (A) rapid boom operation under horizontal crown motion and (B) boom inching operation under saturated condition of full lever of arm and bucket.

For the case (A), from the state that pump pressure is dependent on light load (arm or bucket), system changes new state suddenly, that is, state that pump pressure is dependent on high boom pressure.
At the same time, oil suddenly flows into high load boom cylinder, and PCs for both heavy and light load port are going throttle their openings, simultaneously.

In this time, control system is governed by pump regulator, heavy load side PC and boom load. Therefore heavy load system tends to become unstable, by its own characteristics.

Reproduced phenomena of this state by simulator are shown in fig.11 (a)(b).

Fig.(a) shows the case of conventional PC $\beta=0$, in other words, flat $P-Q$ curve. Boom pressure is observed hunting in this case.

Fig. (b) shows the case of damped PC, $\beta<0$. Boom hunting is suppressed fairly well.

In case of (B), pump delivery pressure is dependent on light load arm or bucket pressure when arm and bucket under saturated operation. From this state, to operate high pressure boom, LS pressure decreases at first, then PC for light load is going to throttle their opening and oil becomes to flow into boom cylinder.

At the same time, pump pressure tends to depend on heavy load side's boom pressure, PCs for light load side are throttling themselves further in proportion to pump pressure rise.

Finally, heavy load side PC becomes full opening, and control system will be governed by pump regulator and light load PC. It dominates stability.

Generally speaking, PC shows even more right side downward flow curve versus differential pressure by the action of flow force at large flow rate domain, Pc designed $\beta=0$, tends to show instability.

Literature

(1) K. Ichiryu : Recent trend and future forecast of hydraulic systems and control, 9 AFK, P345, B2( 1990)

(2) K. Warren : Efficient and flexible hydraulic system for mobile equipment : 7AFK, P325, B2( 1986)

(3) P. Krus : On load sensing fluid power systems, dissertation of Linkoping $\mathrm{TH}(1988)$ 